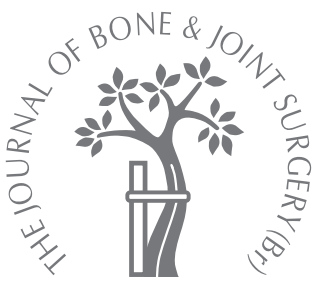

\title{
Three-dimensional CT analysis to determine acetabular retroversion and the implications for the management of femoro-acetabular impingement
}

\author{
W. Dandachli, \\ S. Ul Islam, \\ M. Liu, \\ R. Richards, \\ M. Hall-Craggs, \\ J. Witt
}

From University College London Hospitals, London, England

\begin{abstract}
This study examined the relationship between the cross-over sign and the true threedimensional anatomical version of the acetabulum. We also investigated whether in true retroversion there is excessive femoral head cover anteriorly. Radiographs of 64 hips in patients being investigated for symptoms of femoro-acetabular impingement were analysed and the presence of a cross-over sign was documented. CT scans of the same hips were analysed to determine anatomical version and femoral head cover in relation to the anterior pelvic plane after correcting for pelvic tilt. The sensitivity and specificity of the cross-over sign were $\mathbf{9 2} \%$ and $\mathbf{5 5 \%}$, respectively for identifying true acetabular retroversion. There was no significant difference in total cover between normal and retroverted cases. Anterior and posterior cover were, however, significantly different $(p<0.001$ and 0.002$)$. The cross-over sign was found to be sensitive but not specific. The results for femoral head cover suggest that retroversion is characterised by posterior deficiency but increased cover anteriorly.
\end{abstract}

Femoro-acetabular impingement has only recently been widely recognised as a cause of early osteoarthritis of the hip, although it was first described by Smith-Petersen over 70 years ago. ${ }^{1}$ More recent interest has been attributed to Stulberg et al, ${ }^{2}$ who recognised a pistol-grip deformity of the femoral head that appeared to predispose to early osteoarthritis. It was, however, Beck et $\mathrm{al}^{3}{ }^{3} \mathrm{Ganz}$ et $\mathrm{al}^{4}$ and Ito et $\mathrm{al}^{5}$ who clarified the morphological appearance of the hip in patients with impingement and put forward a theory on how the joint becomes damaged in these patients. Two main types of impingement have been described. Cam impingement occurs where there is a reduced femoral head/neck offset, and pincer impingement where the abnormality is primarily on the acetabular side as a result of excessive cover of the femoral head by the acetabulum, such as in acetabular retroversion and protrusion. A significant proportion of patients presenting for treatment of femoroacetabular impingement are considered to have a combination of these types of impingement. The diagnosis of a cam lesion can be made on plain radiographs, MRI and CT, ${ }^{5,7-9}$ and is relatively straightforward, although assessment of the magnitude of the deformity may be subject to problems of reproducibility. ${ }^{8}$

The diagnosis of acetabular retroversion is more difficult, largely because it is determined by visualising the relationship between the anterior and posterior walls and determining the presence of a cross-over sign and posterior wall sign on a plain pelvic radiograph. ${ }^{10}$ This can be greatly influenced by the quality of the radiograph and the degree of pelvic tilt, ${ }^{11-13}$ raising the question of the reliability of this sign in determining true anatomical version of the acetabulum. This determination is further complicated by the version of the acetabulum changing from cranial to caudal to become naturally increasingly anteverted. ${ }^{14,15}$ What determines excessive retroversion is hard to define.

We used a CT technique that allows standardised three-dimensional (3D) analysis of acetabular inclination and anteversion and calculation of femoral head cover in relation to a specific anatomical reference plane (the anterior pelvic plane) ${ }^{16}$ to look at the relationship between radiological interpretation of retroversion and objective values obtained by CT. The aim of this study was to look at the relationship between the extent of the cross-over sign and the posterior wall sign from plain pelvic radiographs compared with the degree of retroversion measured by $3 \mathrm{D}$ CT. In addition, we wanted to determine whether, in the case of true retroversion of the acetabulum, there was indeed increased cover of the femoral head anteriorly compared with normal data, and how this related to the degree of posterior cover. 


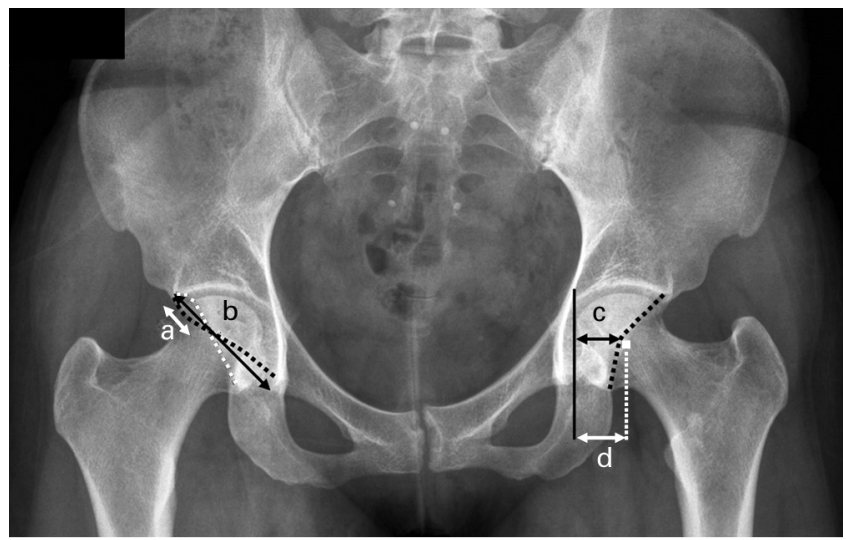

Fig. 1

Anteroposterior pelvic radiograph showing the methods of measuring the cross-over and posterior wall distances. On the right hip, the distance to cross-over (distance a) is expressed as a percentage of the acetabular diameter (distance b). On the left hip, the distance between the posterior wall and the medial edge of the femoral head (distance $c$ ) is expressed as a percentage of the radius of the femoral head (distance $d$ ).

\section{Patients and Methods}

Plain pelvic radiographs of 33 consecutive patients being investigated for symptoms suggestive of femoro-acetabular impingement were analysed. Their mean age was 28 years ( 15 to 45$)$. One patient had already undergone a unilateral hip resurfacing arthroplasty and another had undergone a unilateral peri-acetabular osteotomy, so there were 64 hips available for analysis, of which 43 were in men and 21 in women. The presence of a cross-over sign was documented. For the hips exhibiting a positive crossover sign, the extent of cross-over was measured by noting the point at which the anterior wall crossed the line of the posterior wall. The distance from the superolateral edge of the acetabulum to the point of cross-over was measured and expressed as a percentage of the total diameter of the acetabulum (Fig 1). ${ }^{11}$ The posterior wall sign was analysed in a similar fashion. The point at which the posterior wall crossed a horizontal line extending from the medial edge of the femoral head to its centre was expressed as a percentage of that distance (Fig. 1). The presence of an associated cam-type abnormality of the femoral head was also documented.

CT scans of the same patients were analysed. The scans were acquired with the Siemens Sensation 64 scanner (Siemens, Erlangen, Germany), and the scanning protocol involved scanning the pelvis in two separate segments, with $1.5 \mathrm{~mm}$ slices at the level of the anterior superior iliac spine and $1 \mathrm{~mm}$ slices across the acetabula. Positioning of patients in the scanner was standardised with the legs in neutral abduction/adduction and with the patellae pointing directly upwards.

We have previously described the 3D CT method for measuring anatomical version, inclination and cover of the

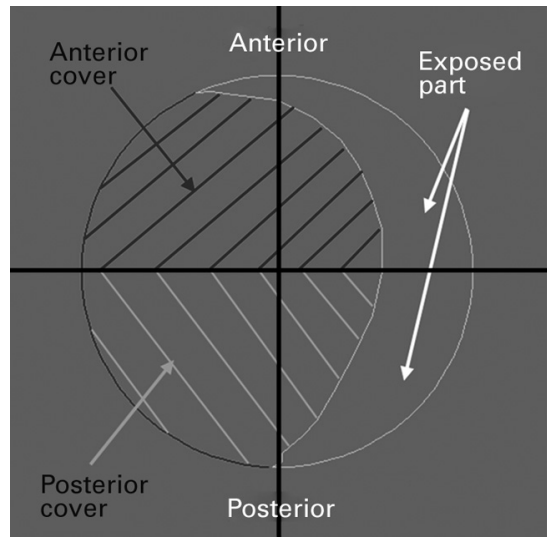

Fig. 2

Topographical image, showing acetabular cover of the femoral head. The circle represents the femoral head and the shaded areas correspond to the part of the femoral head covered by the acetabulum. femoral head. ${ }^{16}$ The analysis is performed in relation to the anterior pelvic plane after correcting for pelvic tilt. In addition to total cover described in the earlier paper, the method has been extended to account for measurement of cover in each of the quadrants representing the femoral head. It was therefore possible to measure anterior and posterior cover separately (Fig. 2).

In order to define true 3D retroversion, the mean acetabular version from our previous study on CT scans of 36 normal hips in subjects with a mean age of 31 years (15 to 61 ) was used. ${ }^{16}$ This mean version angle minus the $95 \%$ confidence interval (mean $-95 \% \mathrm{CI}=14.2^{\circ}$ ) was taken as the limit below which true retroversion was defined. Using this criterion, the 64 hips in the impingement group were classified as anteverted or retroverted. The results were compared with the classification provided by the presence or absence of the cross-over sign and posterior wall sign on the plain radiographs. The sensitivity, specificity, and positive and negative predictive values for these two radiological signs were calculated.

Statistical analysis. In the cases with a positive cross-over sign, the relationship between the distance to cross-over and 3D version was analysed and Pearson's correlation coefficient was calculated using SPSS statistical software (SPSS Inc., Chicago, Illinois). The relationship between the femoral-head-centre-to-posterior-wall distance and 3D version in all of the hips in the impingement group was also studied by calculating Pearson's correlation coefficient.

Finally, the $t$-test assuming unequal variances was used to compare acetabular cover of the femoral head in the anteverted subgroup with that in the truly retroverted subgroup. This was done for total, anterior and posterior cover individually, as well as for the anterior-to-posterior cover 
Table I. Acetabular version for the 64 hips studied, as determined by the cross-over sign, the posterior wall sign, and the 3D CT method

\begin{tabular}{lcc}
\hline & \multicolumn{2}{l}{ 3D CT method } \\
\cline { 2 - 3 } & Retroverted & Anteverted \\
\hline $\begin{array}{l}\text { Cross-over sign } \\
\quad \text { Positive }\end{array}$ & 24 & 17 \\
$\quad$ Negative & 2 & 21 \\
Posterior wall sign & & \\
$\quad$ Positive & & 18 \\
$\quad$ Negative & 21 & 20 \\
\hline
\end{tabular}

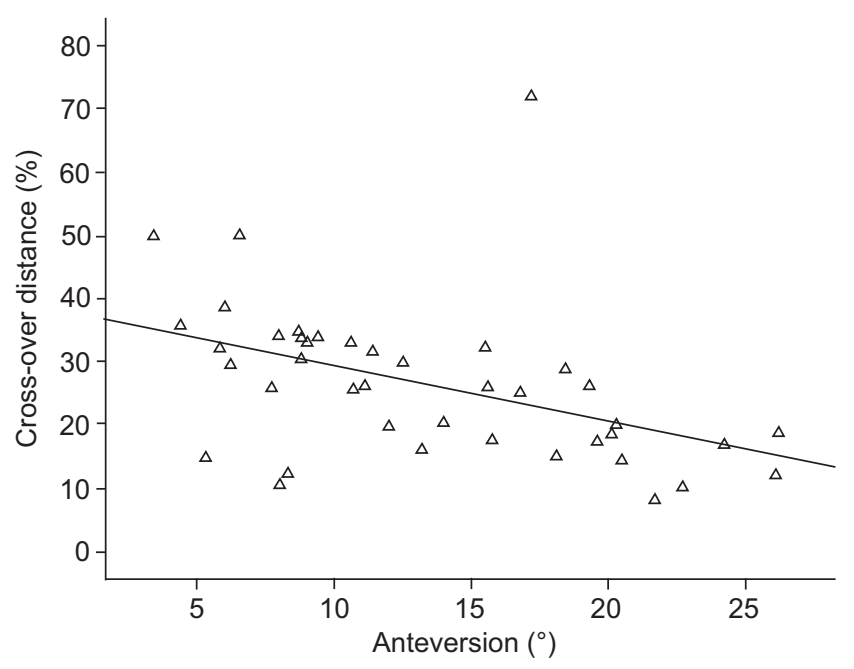

Fig. 3

The relationship between the cross-over distance (as a percentage of the acetabular diameter) and 3D acetabular version.

ratio. A p-value $<0.05$ was indicative of a statistically significant difference.

\section{Results}

Of the 64 hips investigated, $41(64 \%)$ had a positive crossover sign on the plain radiographs. Of these, only 24 hips were found to have true acetabular retroversion. Of 26 truly retroverted acetabula only two had a negative cross-over sign (Table I). The sensitivity, specificity and positive and negative predictive values for the cross-over sign were $92 \%, 55 \%$, $59 \%$ and $91 \%$, respectively, whereas those for the posterior wall sign were $81 \%, 53 \%, 54 \%$ and $80 \%$, respectively.

For the hips with a positive cross-over sign, the crossover distance (as a percentage of the entire length of the lateral acetabular opening) was correlated with $3 \mathrm{D}$ version $(\mathrm{r}=-0.45, \mathrm{p}=0.01)$ (Fig. 3). For the cases with a positive posterior wall sign, the femoral-head-centre-to-posteriorwall distance (as a percentage of the femoral head radius) was also correlated with $3 \mathrm{D}$ version $(\mathrm{r}=0.38, \mathrm{p}=0.05)$ (Fig. 4).

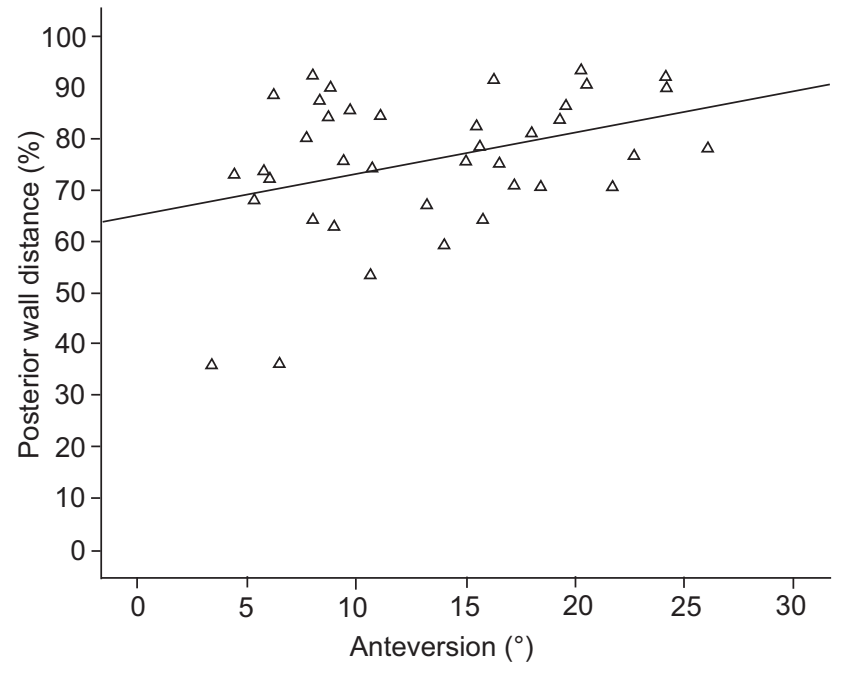

Fig. 4

The relationship between the femoral-head-centre-to-posterior-wall distance (as a percentage of the femoral head radius) and 3D acetabular version.

The results for acetabular cover of the femoral head are summarised in Table II. Using the $14.2^{\circ}$ cut off for the retroversion, there were 38 anteverted and 26 retroverted hips. The mean version in the anteverted subgroup was $20.7^{\circ}$ (SD $4)$, whereas in the retroverted subgroup it was $9^{\circ}(\mathrm{SD} 3)(\mathrm{p}<$ $0.001)$. There was no statistically significant difference in total cover of the femoral head between the anteverted and the truly retroverted subgroups $(71 \%$ vs $72 \%$, respectively; $\mathrm{p}=0.55)$. Anterior cover was significantly higher $(35 \%$ vs $32 \% ; \mathrm{p}=0.001)$, but posterior cover was significantly lower $(37 \%$ vs $39 \%$; $\mathrm{p}=0.002)$ in the retroverted subgroup. The anterior/posterior cover ratio was significantly higher in the retroverted group (0.95 vs 0.83 ; $\mathrm{p}<0.001$ ).

There was a cam deformity of the femoral head in 37 of the 64 hips $(58 \%)$ and of these, $14(38 \%)$ were associated with true retroversion. Conversely, of 26 hips with retroversion, $14(54 \%)$ had an associated cam deformity.

\section{Discussion}

Acetabular retroversion represents a structural abnormality that can be responsible for femoro-acetabular impingement. Its diagnosis has traditionally been made by the presence of a cross-over sign on plain pelvic radiographs. ${ }^{10}$ The presence or absence of this sign may be difficult to interpret. Moreover, pelvic tilt may have a profound effect on the relationship between the anterior and posterior walls. $^{12,13}$ We have used a 3D CT technique that allows standardised analysis of acetabular orientation in relation to a specific reference plane after correcting for pelvic tilt. It was then possible to define true $3 \mathrm{D}$ retroversion and test the reliability of the cross-over sign.

Although the cross-over sign was found to be sensitive enough to identify $92 \%$ of the retroverted cases, its 
Table II. The total, anterior and posterior cover, as well as anterior/posterior cover ratios, for the anteverted and retroverted subgroups

\begin{tabular}{lllll}
\hline & Total cover (\%) & Anterior cover (\%) & Posterior cover (\%) & Anterior/posterior cover ratio \\
\cline { 2 - 5 } Subgroup & Mean (SD) & Mean (SD) & Mean (SD) & Mean (SD) \\
\hline Anteverted & $71.4(4.6)$ & $32.3(2.6)$ & $39.1(2.5)$ & $0.83(0.05)$ \\
Retroverted & $72.1(5.0)$ & $35.1(2.8)$ & $37.0(2.5)$ & $0.95(0.07)$ \\
p-value & 0.55 & $<0.001$ & 0.002 & $<0.001$ \\
\hline
\end{tabular}

specificity was low $(55 \%)$, with just under half of the anteverted cases being wrongly labelled as retroverted. Of the 64 hips analysed, there were 17 false positives, where cases that were truly anteverted were labelled as retroverted because of the presence of a cross-over sign. This is in contrast to the study by Jamali et al, ${ }^{11}$ where it was found that the cross-over sign had a positive predictive value of $90 \%$ and a specificity of $95 \%$. Their study was based on pelvic specimens from a collection of skeletons which had anatomical measurements made and which were then correlated with radiographs of the same specimens. All measurements were made with the position of the pelvis standardised to the anterior pelvic plane.

Similarly, the posterior wall sign had a high sensitivity, with only five false negatives, but it lacked specificity, giving rise to 18 false positives out of the 64 hips. Of the 17 false positives related to the cross-over sign, four hips had a negative posterior wall sign. Therefore, combining the posterior wall sign with the cross-over sign still gave 13 false positives or a specificity of $66 \%$.

In reality, any consecutive series of patients attending for radiographs of their pelvis will exhibit varying degrees of pelvic tilt. This was highlighted in the study by Siebenrock et al, ${ }^{13}$ where 86 plain pelvic radiographs were analysed and compared with a cadaver model. The distance from the sacrococcygeal joint to the symphysis pubis was measured and ranged from $8 \mathrm{~mm}$ to $50 \mathrm{~mm}$ in men and $15 \mathrm{~mm}$ to $72 \mathrm{~mm}$ in women. This corresponded to $12^{\circ}$ reclination to $9^{\circ}$ inclination in men and $8^{\circ}$ reclination to $9^{\circ}$ inclination in women. A pelvis was considered to be in neutral when the distance between the sacrococcygeal joint and the symphysis pubis was $32 \mathrm{~mm}$ in men and $47 \mathrm{~mm}$ in women. In order to attempt correction for the tilt and rotation effects of plain radiological analysis, Tannast et $\mathrm{al}^{12}$ developed a computer-assisted method to allow analysis of digitised radiographs correcting for these variables. They found that the distance from the symphysis pubis to the sacrococcygeal joint was not sufficiently accurate to determine the degree of pelvic tilt, and a lateral radiograph was required to calibrate the system.

The frequency with which pelvic tilt affects interpretation of radiographs is well illustrated in the study by Kalberer et al, ${ }^{17}$ in which they report a new sign denoting acetabular retroversion based on the projection of the ischial spine into the pelvis. In their study, 1010 pelvic radiographs were reviewed in patients being assessed for a painful hip. In order to standardise their analysis only those radiographs denoting neutral rotation of the pelvis were included. Further exclusion of radiographs with inadequate exposures left only 149 of the original 1010 (14.7\%) for analysis.

In our study we did not attempt to make measurements between the sacrococcygeal joint and the pubic symphysis, as all the radiographs were taken in a standard fashion with the patient consistently positioned, and were considered satisfactory in terms of rotation. However, it was accepted that there would be variation in terms of pelvic tilt. Our findings with regard to the reliability of the cross-over sign in determining the presence of significant retroversion are almost certainly due to this variable pelvic tilt, and illustrate how this radiological sign can be misleading.

The question whether acetabular retroversion is associated with excessive cover anteriorly or reduced cover posteriorly has had only limited investigation. Giori and Trousdale $^{18}$ investigated the appearance of a model acetabulum which had the anterior wall of the acetabulum augmented or the posterior wall made deficient, and found that the acetabulum with a deficient posterior wall matched radiographs of the pelvis with a typical cross-over sign most accurately. They also found that retroversion was observed more commonly $(20 \%)$ on radiographs of patients with osteoarthritis of the hip, compared with radiographs taken for non-orthopaedic conditions ( $5 \%$ ), but no comment was made regarding an assessment of the degree of pelvic tilt in these cases. This was similar to the findings of Ezoe, Naito and Inoue, ${ }^{19}$ who excluded radiographs considered to exhibit excessive pelvic tilt, where $6 \%$ of normal hips and $20 \%$ of those with osteoarthritis showed evidence of a cross-over sign.

Whether acetabular retroversion is associated with excessive femoral head cover or represents part of a continum of acetabular orientation is important in the understanding of how this morphology may or may not be implicated in producing problems of impingement in the hip. This prompted us to measure the degree of cover of the femoral head anteriorly and compare it with the degree of cover posteriorly. Our findings showed that the percentage cover of the femoral head anteriorly in retroverted hips was higher than that seen in hips with normal anteversion, and that the degree of posterior cover was significantly lower. This was illustrated by the anterior/posterior cover ratio, which was significantly different between the two groups. 
Another important finding was that the total femoral head cover was not excessive and did not differ significantly between the two groups. The degree of femoral head cover fell within the range reported in our previous study on normal hips, where the mean was $73 \%$ (95\% CI 71 to 74$).{ }^{16}$

There is a perception that acetabular retroversion is associated with excessive cover of the femoral head by the acetabulum anteriorly, and has in part given rise to the concept of removing part of the anterior wall of the acetabulum as the treatment. ${ }^{6,20}$ Removing bone from the anterior part of the acetabulum to normalise the radiological relationship of anterior to posterior walls in such cases may have significant implications in terms of joint contact pressures. It has been suggested that up to $1 \mathrm{~cm}$ of acetabulum can be excised to remove the chondral lesions associated with impingement. ${ }^{20}$ This strategy in a hip with normal total femoral head cover at the outset may not be optimal in the long term. Our findings would tend to support the concept of acetabular reorientation being a more appropriate treatment. ${ }^{10,21}$

The exact mechanism whereby retroverted hips may be more predisposed to the development of osteoarthritis is difficult to determine. It is known that the posterior aspect of the acetabulum is subjected to high loads during activities of daily living. ${ }^{22-24}$ One could hypothesise that the mechanism for the development of osteoarthritis in these cases has more to do with higher joint contact pressures as a result of relative posterior dysplasia ${ }^{18}$ than to the so-called contre coup lesion, where the femoral head is thought to be levered posteriorly as a result of impingement anteriorly. ${ }^{4}$ In some circumstances it is possible that there is a combination of these effects contributing to the development of arthritis, depending on the degree of retroversion.

The frequency with which a cam lesion is combined with acetabular retroversion is much lower in our series than in other reports in the literature. In a series of 26 hips undergoing periacetabular osteotomy for acetabular retroversion, Siebenrock et $\mathrm{al}^{21}$ found that 24 had evidence of a reduced femoral head/neck offset which was corrected at surgery. In the series reported by Espinosa et $\mathrm{al}^{6}{ }^{6}$ in which 60 hips underwent surgery for cam impingement, 25 had resection of the acetabular rim and labrum and 35 had resection followed by re-fixation of the labrum, which suggests that all their cases were treated as combined pincer and cam. The indication for intervention on the acetabular side was described as relative acetabular overgrowth with the amount of rim resected determined by the extent of over-coverage and the magnitude of damage to the acetabular cartilage. This appeared to be based on an intra-operative assessment. No pre-operative data on these patients were provided to determine how acetabular cover was assessed, and no information was provided on the proportion of patients exhibiting a pre-operative cross-over sign.

We found that $38 \%$ of cases with a cam deformity were associated with true acetabular retroversion, and that $54 \%$ of the hips identified as being retroverted had an associated cam deformity of the femoral head. These proportions would change depending on the threshold applied to the definition of retroversion. We considered it appropriate to use CT reference data from normal hips in defining the level at $14.2^{\circ}$. We do not know of any other attempts to define this value. Of note, in our previous study on normal hips, the degree of retroversion ranged between $12.5^{\circ}$ and $0.5^{\circ}$, by which 12 of the 36 hips had retroversion as determined by our definition. ${ }^{16}$ The proportion was higher than in other studies based on plain radiographs, and suggests a considerable range of normal acetabular version. ${ }^{18,19}$ The study by Maruyama et $\mathrm{al}^{14}$ looking at pelvic skeletons found mean acetabular version to be $19.9^{\circ}$, with a range between $7^{\circ}$ and $42^{\circ}$ when acetabular version was measured at the centre of the acetabulum along the anterior to posterior ridges. That method would tend to increase the degree of anteversion slightly compared with the way our analysis was performed.

Determining whether a hip with relative acetabular retroversion is producing symptoms of impingement may be difficult, particularly in the presence of an associated cam lesion. Our study did not take into account the version of the femoral neck, which is likely to affect whether or not a hip becomes symptomatic. Tonnis and Heinecke ${ }^{25}$ looked at the relationship between femoral and acetabular version in a large number of patients and were able to divide the hips studied into nine combinations of femoral and acetabular version. It was found that $31 \%$ had a combination of decreased femoral and acetabular anteversion, $21 \%$ had increased acetabular anteversion in combination with decreased femoral anteversion, $20 \%$ had normal acetabular anteversion in combination with decreased femoral anteversion, and $9 \%$ had decreased acetabular anteversion compensated for by increased femoral anteversion.

Publications on the treatment of femoro-acetabular impingement will be difficult to interpret unless a standardised way of assessing the morphology of a hip is established. It is too simplistic to consider the presence of a crossover sign on a radiograph as evidence that a hip has pincer impingement. CT analysis has advantages, as the variables associated with radiological analysis are removed. We have found that radiological assessment of anatomical retroversion is not accurate, and that acetabular retroversion is associated with increased anterior cover of the hip and decreased posterior cover. Our CT investigation has indicated that there may be a lower incidence of true acetabular retroversion in association with a cam lesion than has been previously reported, but the retroverted acetabula have quite a high incidence of associated cam lesions. The total femoral head cover is not increased in these cases, and this should be taken into consideration when deciding on the appropriate surgical intervention.

We feel that, as well as clearly defining acetabular anatomy and orientation, 3D CT evaluation allows detailed analysis of femoral head topography, which is of value when mini-open or arthroscopic techniques are used for treatment. Modern scanning protocols allows this to be achieved with low levels of radiation exposure. ${ }^{16,26}$ 
No benefits in any form have been received or will be received from a commercial party related directly or indirectly to the subject of this article.

\section{References}

1. Smith-Petersen MN. Treatment of malum coxae senitis, old slipped upper femoral epiphysis, intrapelvic protrusion of the acetabulum, and coxa plana by means of acetabuloplasty. J Bone Joint Surg 1936;18:869-80.

2. Stulberg SD, Cordell LD, Harris WH, Ramsey PL, MacEwen GD. Unrecognised childhood hip disease: a major cause of idiopathic osteoarthritis of the hip. In: Amstutz HC, ed. The Hip. Procs Third Open Scientific Meeting of the Hip Society. St Louis: CV Mosby, 1975:212-28.

3. Beck M, Kalhor M, Leunig M, Ganz R. Hip morphology influences the pattern of damage to the acetabular cartilage: femoroacetabular impingement as a cause of early osteoarthritis of the hip. J Bone Joint Surg [Br] 2005;87-B:1012-18.

4. Ganz R, Parvizi J, Beck M, et al. Femoroacetabular impingement: a cause for osteoarthritis of the hip. Clin Orthop 2003;417:112-20.

5. Ito K, Minka MA 2nd, Leunig M, Werlen S, Ganz R. Femoroacetabular impingement and the cam-effect: a MRI-based quantitative anatomical study of the femoral head-neck offset. J Bone Joint Surg [Br] 2001;83-B:171-6.

6. Espinosa N, Rothenfluh DA, Beck M, Ganz R, Leunig M. Treatment of femoroacetabular impingement: preliminary results of labral refixation. J Bone Joint Surg [Am]2006;88-A:925-35.

7. Meyer DC, Beck M, Ellis T, Ganz R, Leunig M. Comparison of six radiographic projections to assess femoral head/neck asphericity. Clin Orthop 2006;445:181-5.

8. Beaule PE, Zaragoza E, Motamedi K, Copelan N, Dorey FJ. Three-dimensional computed tomography of the hip in the assessment of femoroacetabular impingement. J Orthop Res 2005;23:1286-92.

9. Notzli HP, Wyss TF, Stoecklin CH, et al. The contour of the femoral head-neck junction as a predictor for the risk of anterior impingement. J Bone Joint Surg [Br] 2002;84-B:556-60.

10. Reynolds D, Lucas J, Laue K. Retroversion of the acetabulum: a cause of hip pain. J Bone Joint Surg [Br] 1999;81-B:281-8.

11. Jamali AA, Mladenov K, Meyer DC, et al. Anteroposterior pelvic radiographs to assess acetabular retroversion: high validity of the "cross-over-sign". J Orthop Res 2007;25:758-65.
12. Tannast M, Zheng G, Anderegg C, et al. Tilt and rotation correction of acetabular version on pelvic radiographs. Clin Orthop 2005;438:182-90.

13. Siebenrock KA, Kalbermatten DF, Ganz R. Effect of pelvic tilt on acetabular retroversion: a study of pelves from cadavers. Clin Orthop 2003;407:241-8.

14. Maruyama M, Feinberg JR, Capello WN, D'Antonio JA. The Frank Stinchfield Award: morphologic features of the acetabulum and femur: anteversion angle and implant positioning. Clin Orthop 2001;393:52-65.

15. Anda S, Terjesen T, Kvistad KA. Computed tomography measurements of the acetabulum in adult dysplastic hips: which level is appropriate? Skeletal Radiol 1991;20:267-71.

16. Dandachli W, Kannan V, Richards R, et al. Analysis of cover of the femoral head in normal and dysplastic hips: new CT-based technique. J Bone Joint Surg [Br] 2008;90-B:1428-34

17. Kalberer F, Sierra RJ, Madan SS, Ganz R, Leunig M. Ischial spine projection into the pelvis: a new sign for acetabular retroversion. Clin Orthop 2008;466:677-83.

18. Giori NJ, Trousdale RT. Acetabular retroversion is associated with osteoarthritis of the hip. Clin Orthop 2003;417:263-9.

19. Ezoe $\mathbf{M}$, Naito $\mathbf{M}$, Inoue $\mathbf{T}$. The prevalence of acetabular retroversion among various disorders of the hip. J Bone Joint Surg [Am] 2006;88-A:372-9.

20. Parvizi J, Leunig M, Ganz R. Femoroacetabular impingement. J Am Acad Orthop Surg 2007;15:561-70.

21. Siebenrock KA, Schoeniger R, Ganz R. Anterior femoro-acetabular impingement due to acetabular retroversion: treatment with periacetabular osteotomy. J Bone Joint Surg [Am] 2003;85-A:278-86.

22. Witte H, Eckstein F, Recknagel S. A calculation of the forces acting on the human acetabulum during walking: based on in vivo force measurements, kinematic analysis and morphometry. Acta Anat (Basel) 1997;160:269-80.

23. Pedersen DR, Brand RA, Davy DT. Pelvic muscle and acetabular contact forces during gait. J Biomech 1997;30:959-65.

24. Hodge WA, Carlson KL, Fijan RS, et al. Contact pressures from an instrumented hip endoprosthesis. J Bone Joint Surg [Am] 1989;71-A:1378-86.

25. Tonnis D, Heinecke A. Acetabular and femoral anteversion: relationship with osteoarthritis of the hip. J Bone Joint Surg [Am] 1999;81-A:1747-70.

26. Henckel J, Richards R, Lozhkin K, et al. Very low-dose computed tomography for planning and outcome measurement in knee replacement: the imperial knee protocol. J Bone Joint Surg [Br] 2006;88-B:1513-18. 